\title{
Bronchoscopy and the chest X-ray
}

\author{
W. HATELY* \\ M.B., M.R.C.P.E., F.F.R.
}

Peter Jones

M.V.O., F.R.C.S.

\author{
BASIL STRICKLAND \\ F.R.C.P., F.F.R., F.A.C.R. \\ Westminster Hospital, London, S.W.1
}

\section{Summary}

Fifty-seven cases of primary bronchogenic carcinoma with X-ray changes, but with negative bronchoscopies, have been reviewed to illustrate the limitations of this method of examination. The reasons for these limitations are discussed.

\section{Introduction}

An increasing number of diagnostic X-ray departments are opening their doors for direct referral of patients from general practitioners without the intervention of a clinical consultant before the patient is radiographed. Communication between diagnostic radiologist and practitioner is direct and hence increased responsibility falls upon both. With this in mind we are concerned with two associated factors:

(1) The delay in referral for brochoscopy when an opacity of doubtful aetiology is found to be present on the X-ray film.

(2) The proper value of bronchoscopy in its relationship to the X-ray appearances.

We wish to emphasize that the two investigations are rarely mutually exclusive but most often complementary.

Linked with the X-ray film, bronchoscopy is the most important single method of reaching a positive diagnosis in neoplasms of the bronchial tree and it is regrettable that many clinicians are so reluctant to refer patients for this simple investigation when an unsuspected opacity is discovered on the X-ray film. The bronchoscope covers a wide area of vision in the proximal bronchial tree (Fig. 1) and diagnosis is often immediate and absolute.

On occasion, however, malignant bronchogenic tumours lie in the vicinity of the major bronchi (as judged by the X-ray film) and yet are not visible at bronchoscopy. Such a negative bronchoscopy will not deter the chest physician from making a diagnosis of malignant disease, but to those unused to the limitations of this otherwise invaluable diagnostic procedure there is a tendency to assume that a negative bronchoscopy renders suspicious X-ray findings less sinister and final diagnosis is corre-

\footnotetext{
* Present address: The London Hospital, London, E.1.
}

spondingly delayed. In this way an operable tumour may be rendered inoperable by the unnecessary passage of time.

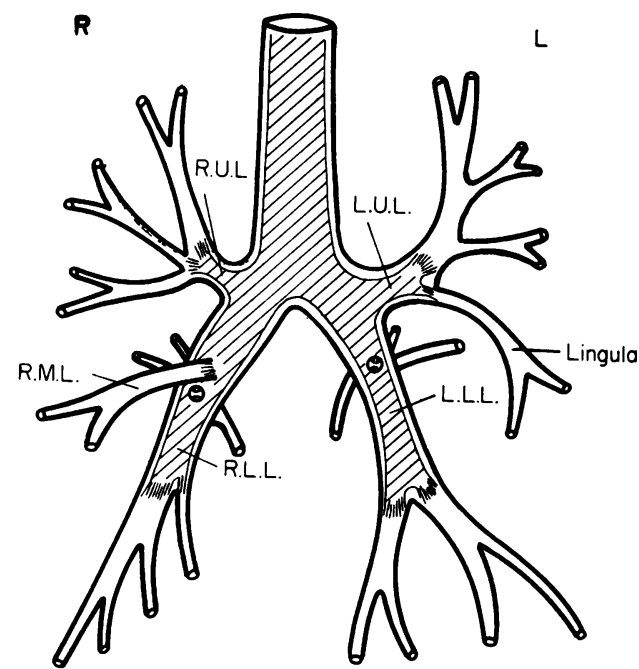

FIG. 1. The bronchial tree. Only the hatched area can be seen routinely at bronchoscopy. A varying degree of visualization is seen in the localized areas marked by short lines.

With this in mind and in an effort to bring these facts to a wider medical audience (outside the field of the chest specialist) we have assessed the site of origin and X-ray appearances of fifty-seven cases of primary bronchogenic carcinoma where bronchoscopy was negative, although it might have been expected that a direct view of the neoplasm could have been obtained through the bronchoscope. In all instances the operator was of sufficient experience to obviate the possibility of an incomplete or inadequate bronchoscopic examination.

\section{Results and discussion}

Study of these fifty-seven cases has shown that there are several reasons for this failure to make the diagnosis by bronchoscopy in the presence of a tumour mass large enough to produce an opacity on chest films taken in both planes, the principal ones being the following: 


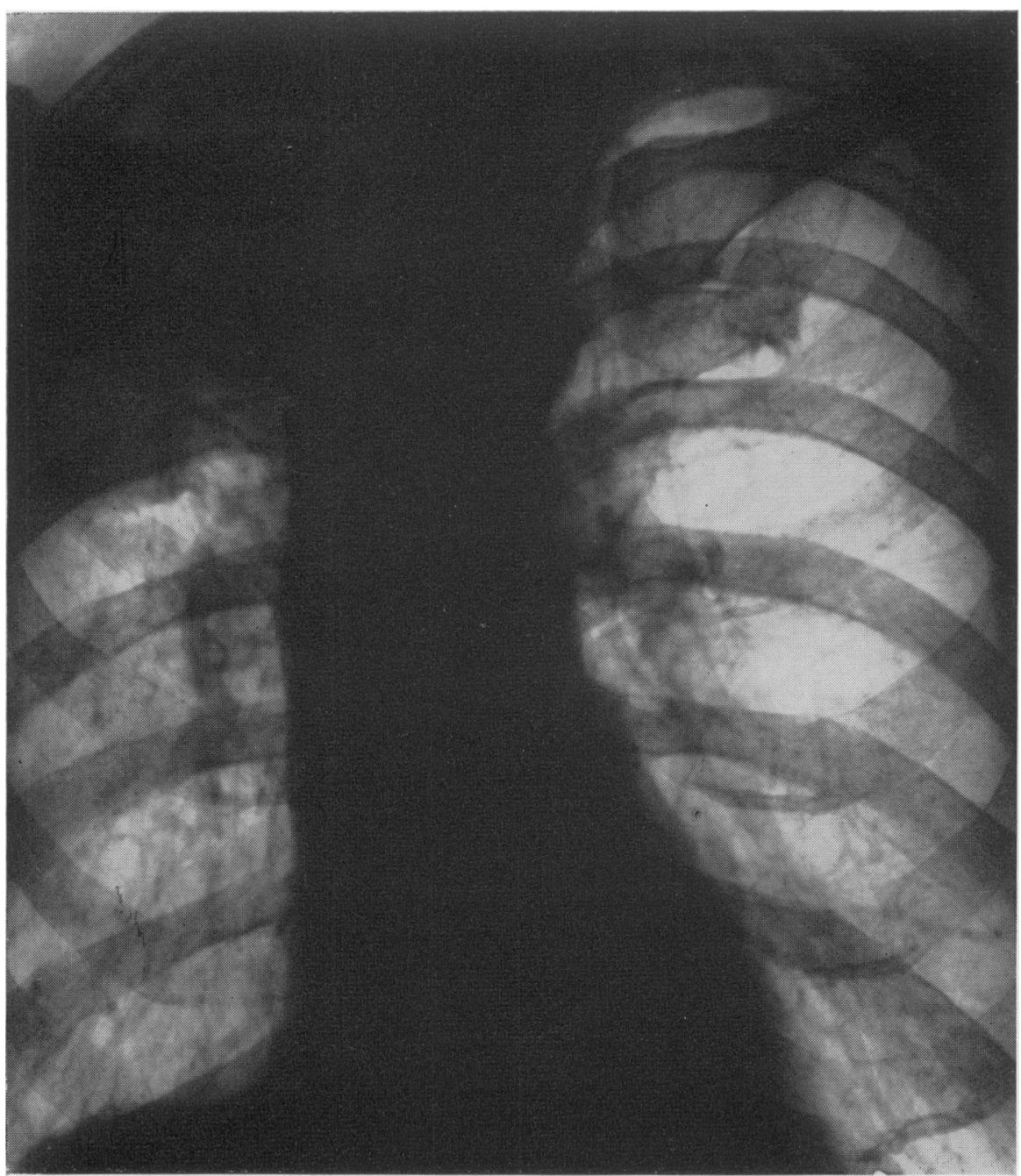

Fig. 2. Carcinoma of the right upper lobe bronchus with lobar collapse-negative bronchoscopy.

(1) Some tumours, even when not situated away from the larger bronchi as judged by the radiographs are unfavourably placed for bronchoscopic vision (an example is illustrated by Fig. 2, where a carcinoma of the right upper lobe bronchus has produced lobar collapse).

(2) Study of Fig. 1 shows that if a tumour obstructs a segmental bronchus it may infiltrate widely giving rise to a large opacity on the radiograph and yet be invisible through the bronchoscope. Six representative examples from our series are illustrated in Fig. 3, depicting the opacities seen in PA and lateral films.

It follows that if a carcinoma presents as collapse of a complete lobe or an entire lung it will usually be seen through the bronchoscope, though this is not always the case since in rare instances a lobe may contract (rather than collapse) by diffuse infiltration by neoplasm and the cause be undetectable on bronchoscopy.

(3) A bronchogenic carcinoma in the peripheral lung field may present as a secondary mass of malignant hilar nodes or as a continuous mass of tumour tissue. This mass may encircle a bronchus without involving its wall and, thus, appear to be well within bronchoscopic vision as judged from the radiograph and yet be invisible at this examination, however experienced the operator (Fig. 5).

(4) Any condition associated with an excess of secretions in the bronchial tree offers a further diffiulty to accurate bronchoscopic diagnosis. In chronic bronchitis not only are the bronchi filled with large amounts of secretion, but the mucosa is 
III

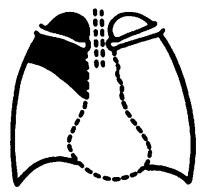

Vil
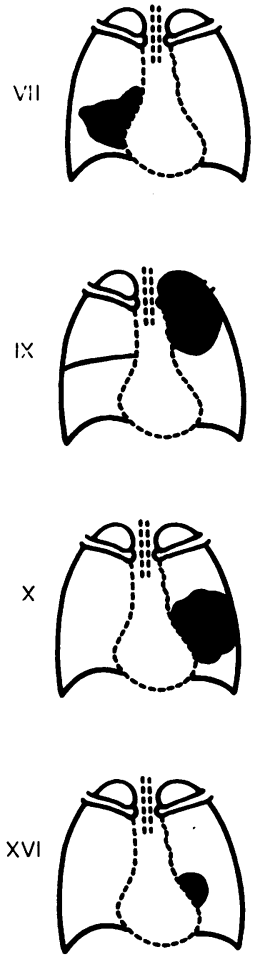

$X L$

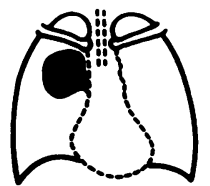

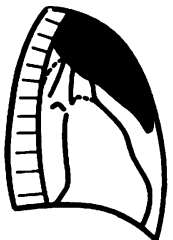
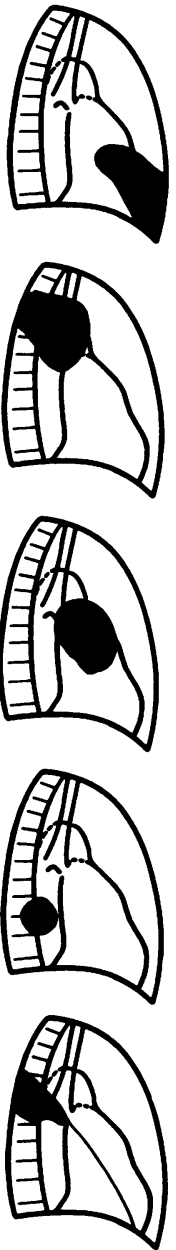

Fig. 3. Drawings of the $\mathrm{X}$-ray findings in six cases of bronchogenic carcinoma where bronchoscopy was negative. The tumour may infiltrate widely to give a large opacity beyond the bronchoscopic vision.

often red and swollen adding an even further limitation to the field of bronchoscopic vision. In theory, bronchial bleeding should aid the siting of an endobronchial neoplasm, but even a small quantity of blood may be dispersed widely throughout the bronchial tree by coughing, confusing the picture and precluding valid diagnosis. In general, bleeding is not helpful in localizing an endobronchial lesion.

Fortunately, an enlarged left atrium, moderate

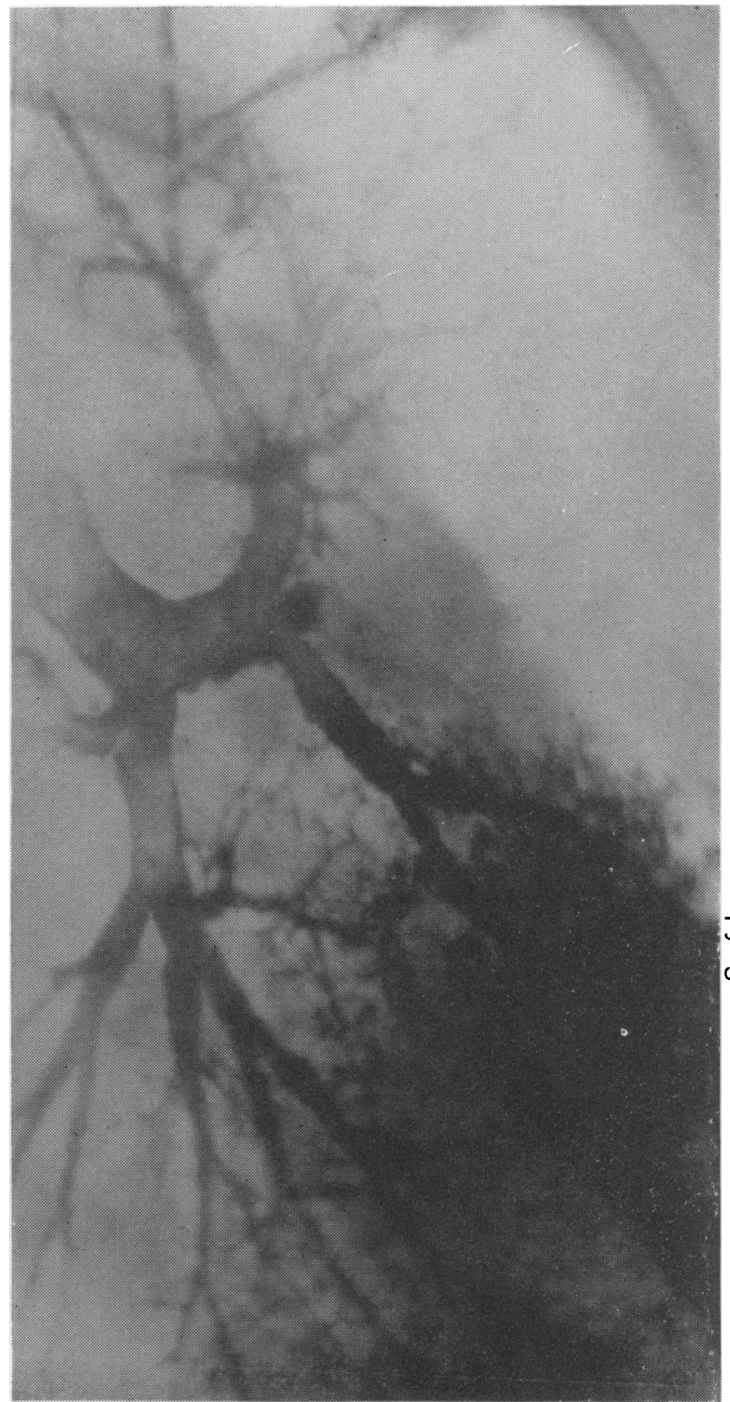

Fig. 4. Carcinoma of the anterior segmental bronchus of the left upper lobe. This was just beyond the area of bronchoscopic vision and bronchography was necessary for accurate localization.

spinal curvature or a calcified aorta do not affect the range of bronchoscopic vision, but aneurysmal dilatation of the aorta may present a partial obstruction to any adequate examination. It goes without saying that all experienced operators will use a telescope in their routine bronchoscopies for only the basal orifices may be seen directly without this instrument. One must be well versed in the normal anatomical variations of the bronchial tree (i.e. the anterior segmental portion of the right upper lobe bronchus may arise from the apical or posterior segmental bronchus), and it must be appreciated that 

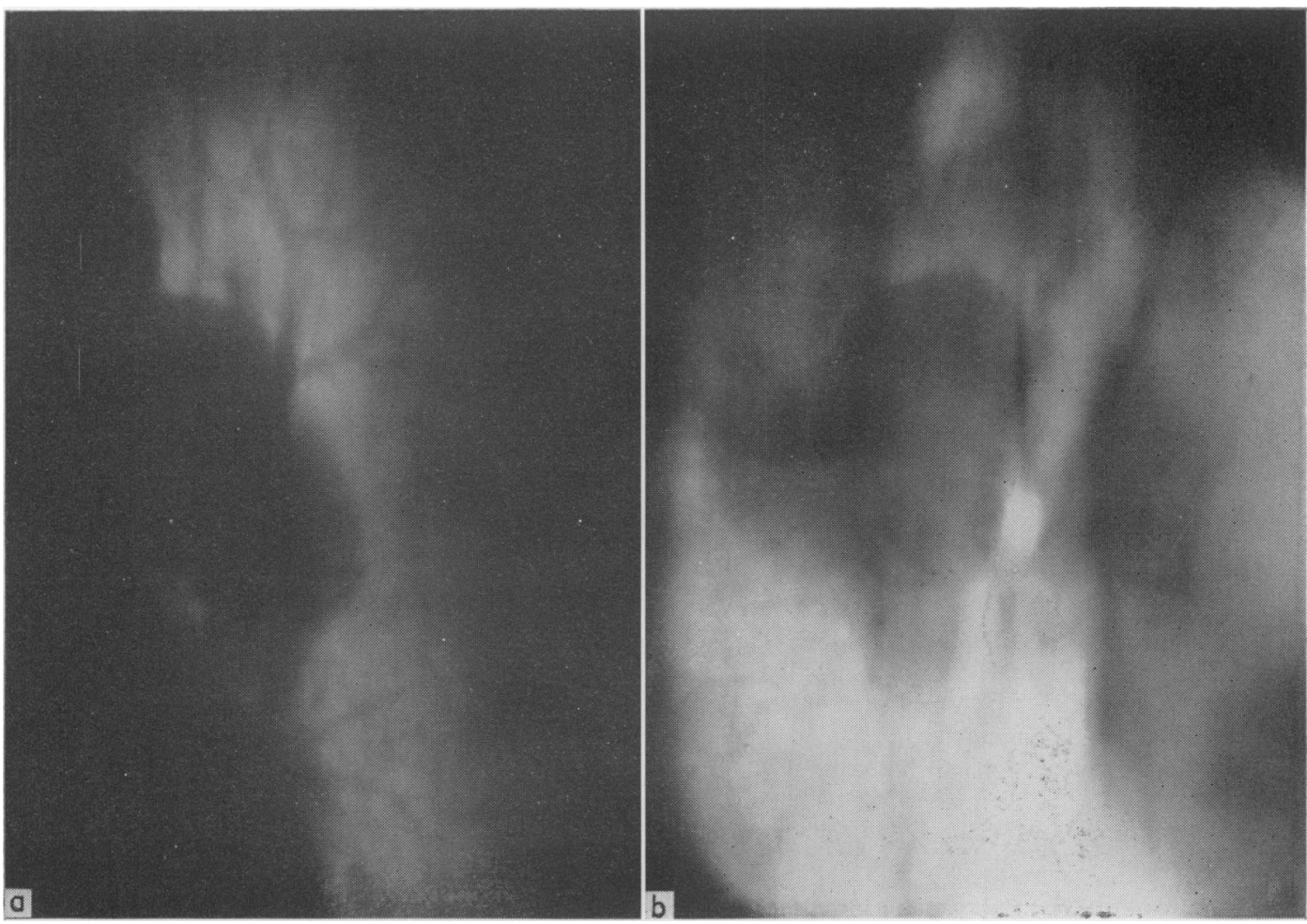

Fig. 5. Carcinoma of the sub-apical region of the left lower lobe. (a) PA, (b) lateral. The malignant mass encircles the bronchus but does not involve the bronchial wall. It is, therefore, not visible at bronchoscopy in spite of its size.

certain bronchial divisions are more difficult to examine than others, e.g. the left upper lobe bronchi in the presence of collapse of the upper and lower lobes-while a varying degree of vision will be obtained of the divisions of the middle lobe bronchus and the apical bronchi of both lower lobes with their segmental divisions.

An awareness of all these and other pitfalls does not preclude major errors which are beyond the control of the bronchoscopist for the reasons outlined at the beginning of this discussion (the expert operator is aware of the limitations set upon him by his 'tools of trade'). An insuffizient awareness of this limitation on the part of the referring physician is fraught with hazard born of a false sense of security.

In any patient where the bronchoscopy is negative but the radiograph 'suspicious' at least six specimens of sputum should be examined microscopically for the presence of tumour cells. The examination must be performed by a pathologist well versed in the sputum cytology of lung carcinoma for in this field experience is the very touchstone of sound diagnosis.

Finally, even if bronchoscopy and sputum cytology are negative the investigation of the patient still calls for bronchography to exclude the presence of an endobronchial neoplasm (Figs. 4 and 6).

Only then may the physician feel reasonably

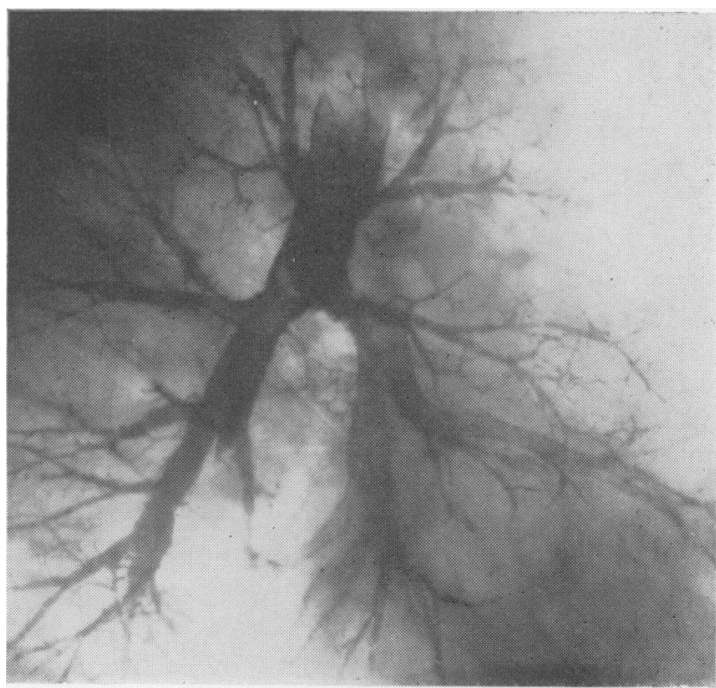

Fig. 6. Carcinoma of the anterior segment of the left lower lobe. The bronchus is occluded. This was not visible at bronchoscopy.

confident that the abnormal opacity on the radiograph is due to a cause other than carcinoma, bearing in mind that all these investigations may be negative in the presence of a peripheral neoplasm. 